\title{
Analysis of the Use of Imipenem at a University Hospital Following the Restructuring of an Antimicrobial Audit System
}

\author{
Souza N.P. ${ }^{1}$, Noblat A.C.B. ${ }^{2}$ and Noblat L. $^{3}$ \\ ${ }^{1}$ Pharmacist, Government Health Department od Bahia; ${ }^{2}$ Physician, Nephrologist, University Hospital Complex "Professor Edgard Santos"; \\ ${ }^{3}$ Associate Professor, Pharmacy School; Federal University of Bahia, Salvador, BA, Brazil
}

\begin{abstract}
This study analyzed the use pattern of imipenem following the restructuring of the antimicrobial audit system at a University Hospital. It was an observational study before and after the restructuring of the antimicrobial audit system in a University Hospital from May to August and then from September to December 2006. The criteria of the rational use of imipenem were obtained from a non-systematic revision of the literature. The collection of data on the general characteristics and clinical state of the patient, the infection and the established therapy was carried out in a previously tested instrument. Data was recorded, revised and analyzed in a database built with the software SPSS ${ }^{\circledR}$ for Windows ${ }^{\circledR}$ PC, version 10.0. The statistical analysis had a descriptive character: frequencies, mean, median and standard deviation. No differences were encountered in relation to the appropriate indication, consumption and clinical outcomes of patients. However, there was a reduction of 4 to $1(75.0 \%)$ in the number of associations with spectrum superposition and an increase of 4 to $8(50.0 \%)$ in the change of therapy. The restructuring of the antimicrobial audit system in the studied hospital did not reflect significantly the increase of the appropriate indication of imipenem. It contributed, however, to the reduction of the inappropriate associations of this antibiotic and to changes of therapy, without, however, compromising the quality of services rendered to patients.

Key-Words: Imipenem, drug use review, drug use evaluation, audit system, antimicrobial policy.
\end{abstract}

Imipenem, a broad spectrum antibiotic, is indicated for the treatment of mixed infections, caused by multiresistant microorganisms, where therapeutic alternatives do not exist [1-3]. However, in the past years, its irrational use grew indiscriminately, elevating the costs of assistance, exposing the patient to a larger number of adverse effects and increasing the incidence of bacterial resistance to this antibiotic, mainly among Gram-negative bacilli that cause nosocomial infections. A study carried out at an academic hospital in Taiwan between 1991 and 2003 demonstrated an increase of 4.2, 5.1 and 801.3 times in the consumption of extended-spectrum cephalosporines, carbapenems and fluoroquinolones, respectively. The elevation in the consumption of cephalosporines and carbapenems demonstrated a significant association with the increase in the resistance of Acinetobacter spp. to meropenem [4]. Another study carried out between 1997 and 2000 reported the association between periods of elevation of the consumption of imipenem and the increase in the resistance of Pseudomonas aeruginosa not only to this antibiotic but also to other beta-lactams such as ceftazidime and piperacilintazobactam [5]. A cohort study showed that, in patients with hospital-acquired bacteremia, the recent use of carbapenems was one of the risk factors for the involvement of extended spectrum beta-lactamases producing (ESBL) enterobacteria (Escherichia coli and Klebsiella spp.) as etiologic agent of the infection. In the prior exposure criterion to antibiotics,

Received on 21 April 2008; revised 29 November 2008.

Address for correspondence: Dr. Lúcia Noblat - Rua Macapá, 461, apt ${ }^{\circ}$ 301, Ed. Mansão Horto de Ondina. Zip code: 40.170.150, Ondina, Salvador, Bahia, Brazil. Financing Source: Unified Health System (SUS).

The Brazilian Journal of Infectious Diseases 2008;12(6):494-498. (C) 2008 by The Brazilian Journal of Infectious Diseases and Contexto Publishing. All rights reserved. only the cephalosporines had a more significant association than the carbapenems in the isolation of bacteria producing ESBL [6].

Among the consequences of indiscriminate use of these drugs, the antimicrobial resistance is a great concern among health professionals, due to the risk of emergence of multiresistant bacteria and superinfections, without a corresponding grImipenem, owth, in the same proportion, in the number of therapeutic alternatives [7-9].

According to the World Health Organization (WHO), the rational use occurs when patients receive medications appropriate to their clinical needs, in doses that meet their own individual requirements, for an adequate period of time, and at the lowest cost to them and their community. The rational use is a global term and encompasses the choice of the drug, its dispensing, its use, the monitoring of the patient's adherence to treatment and follow-up of the desired effects and possible adverse events resulting from the treatment [10]. Five criteria guide appropriate use: clinical evidence-based therapy, therapeutic benefits, safety, costeffectiveness and optimal drug dose and duration in the shorter-course [11]. As a result, when the use of a drug meets the general criteria, the use is considered rational and when the established therapy is the most suitable for a specific patient, the use is considered appropriate $[12,13]$.

One of the strategies for the reduction of the inappropriate antimicrobial use is the evaluation of prescriptions of these drugs by an infectologist member of the Hospital InfectionControl Service, a procedure called antimicrobial audit [14]. A study undertaken in 1991 demonstrated that the removal of this evaluation system contributed to the increase of the inappropriate use of antibiotics. With respect to imipenem, in the therapeutic indication criterion, this increase went from 4/ $42(10.0 \%)$ to $12 / 42(29.0 \%)$ [15]. 
Various studies associate the implementation of audit to the reduction in the inappropriate antimicrobial consumption and to the improvement in the microorganisms' sensibility profile, mainly in the intensive care unit (ICU) [16,17].

Between January and July 2006, the expenses with imipenem corresponded to $25.5 \%$ of the financial resources destined to the acquisition of antimicrobial in the studied hospital. Additionally, in September of that same year, the Hospital Infection-Control Service went back to carry out again the regular antimicrobial audit program. In this context, this study was done with the objective of analyzing alterations in the use pattern of imipenem following the restructuring of the antimicrobial audit system.

\section{Material and Methods}

A "before-after" observational study was carried out in a public, general university hospital, with 269 active beds and considered a reference for the state of Bahia, Brazil.

All the inpatients that used imipenem between May and December 2006 were selected. Those that used between May 1st and September 4th were inserted in the P1 group (before the audit), while the ones that used imipenem starting from September 5th and concluded the therapy up to December 31th were inserted in the P2 group (after the audit). Patients that used the imipenem in both periods were excluded. Untracked or incomplete records were considered as loss.

\section{Use of Antibiotics Policy}

The policy for the use of antibiotics in this hospital establishes that the prescription of these drugs should come along with an antimicrobial control record duly completed, signed and dated by the prescribing physician. This record contains: the patient's identification and clinical diagnostic; topography and origin of the infection; isolated microorganisms and their sensibility profile; indication, administration route, dosage, interval and duration of antibiotics treatment. The pharmacist is responsible for the evaluation of the dose, the interval of usage, the indication of therapy and association of antibiotics. When detecting an inappropriate prescription, this professional should suggest to the prescriber the necessary modification, based on the scientific evidence and the hospital's ecology. More complex situations are discussed with the infectologist before the dispensing of the first dose.

\section{Antimicrobial Audit}

The antimicrobial audit was restructured in September of 2006. The infectologist now evaluates the prescription on a daily basis, suggesting to the prescribers, when necessary, modifications in the therapy and more cost-effective alternatives.

\section{Establishment of Criteria}

The criteria for the analysis of the use of this antibiotic were established through a non-systematic revision of the literature in the databases Medline, Lilacs, Web of Science, Uptodate and Micromedex, using the keywords: imipenem, carbapenems, antibiotic, infectious disease physician, infectious disease consultant, resistance, use, usage, restricted use, audit, evidence-based medicine, practice guideline, clinical protocols, physician's practice patterns, drug use review, critical pathways, review, drug utilization evaluation.

The variables used for the analysis of the use of imipenem were: origin, topography and etiologic agent of the infection; the patient's clinical state; indication, dosage and interval, duration of the therapy and prior use of antibiotics. The clinical aspects analyzed were: immunosuppressive state, beta-lactam hypersensitivity and neurological disease.

The use was considered appropriate in the empirical treatment of severe nosocomial infections, based on the microbiological profile of the institution; after therapeutic failure of the first-choice antibiotics; on the specific treatment of infections where it was the only therapeutic alternative or more cost-effective and on the treatment of infections caused by multiresistant microorganisms [18-22]. For instance, for the treatment of serious infections caused by ESBL-Klebsiella pneumoniae, imipenem is considered the first-choice antibiotic [23]. Two factors justify the use of imipenem, even in the cases of apparent in vitro sensibility to piperacilintazobactam: the risk of therapeutic failure caused by the increase of the minimum inhibitory concentration (MIC) of the antibiotic in the presence of high inoculum concentration (inoculum effect) [24] and the development of bacterial resistance during the therapy [25]. In the cases of infections by enterobacteria sensitive to just amicacin and to imipenem as a result of being a multiresistant bacteria, an antibiotic with a broader spectrum is indicated to the detriment of the monotherapy with amicacin [23].

The association of antibiotics was considered inappropriate when there was an action spectrum superposition.

The comparison of the consumption of imipenem in the two periods was carried out using the international measure DDD/100 beds-day established by the World Health Organization Collaborating Centre for Drug Statistics Methodology [26].

The causes of the alterations in the use were ascertained when analyzing the de-escalation of the therapy (substitution of imipenem following evidence of microbial sensibility to a narrower spectrum antibiotic), adjustment of specific therapy (adequation of therapy that was based from the onset on microbiological results), lacks of drug or the restriction of its use and occurrence of adverse effects or bacterial resistance. The method used for the determination of the in vitro sensibility of the bacteria to the antimicrobial agents is the agar disk-diffusion. The quality control is carried out monthly using the following strains: Pseudomonas aeruginosa ATCC 27853, Staphylococcus aureus ATCC 25923 and Escherichia coli 25922. The results are interpreted based on the criteria of the Clinical and Laboratory Standard Institute (CLSI) [27]. 
Table 1. General characteristics of patients.

\begin{tabular}{lcc}
\hline Characteristics & $\begin{array}{c}\text { P1 (N=25) } \\
\mathbf{N ~ ( \% )}\end{array}$ & $\begin{array}{c}\text { P2 (N=26) } \\
\text { N (\%) }\end{array}$ \\
\hline $\begin{array}{l}\text { Gender } \\
\quad \text { Female }\end{array}$ & $13(52)$ & $15(57.7)$ \\
Ward & & \\
$\quad$ Surgical & $5(20)$ & $5(19.2)$ \\
$\quad$ Medical & $7(28)$ & $10(38.5)$ \\
$\quad$ Infectious diseases & $1(4)$ & $2(7.7)$ \\
$\quad$ Intensive therapy & $12(48)$ & $9(34.6)$ \\
Age (years) & & \\
$\quad$ Mean \pm S.D & $49.5 \pm 22.6$ & $51.8 \pm 19.7$ \\
$\quad$ Median & 52 & 52 \\
Length of stay (in days) & & \\
$\quad$ Mean \pm S.D & $47.9 \pm 31.9$ & $62.2 \pm 38.6$ \\
$\quad$ Median & 44 & 46 \\
\hline
\end{tabular}

Table 2. Infection data.

\begin{tabular}{lcc}
\hline Infection data & $\begin{array}{c}\text { P1 (N=25) } \\
\text { N (\%) }\end{array}$ & $\begin{array}{c}\text { P2 (N=26) } \\
\text { N (\%) }\end{array}$ \\
\hline Origin & & \\
$\quad$ Hospital infection & $24(96 \%)$ & $24(92.3 \%)$ \\
Etiologic agent & & \\
Enterobacteria & $11(44 \%)$ & $13(56.5 \%)$ \\
Gram-negative & $2(8 \%)$ & $7(30.4 \%)$ \\
non-enteric bacilli & & \\
Gram-positive aerobic & $4(16 \%)$ & $2(8.7 \%)$ \\
$\quad$ cocci & & \\
Gram-negative & $1(4 \%)$ & $1(4.3 \%)$ \\
$\quad$ unidentified slender rods & & \\
Topography & & $1(3.8 \%)$ \\
Intra-abdominal cavity & $2(8 \%)$ & $4(15.4 \%)$ \\
Indefinite & $1(4 \%)$ & $3(11.5 \%)$ \\
Skin & $4(16 \%)$ & $9(34.6 \%)$ \\
Systemic & $9(36 \%)$ & $6(23.1 \%)$ \\
Respiratory tract & $4(16 \%)$ & $1(3.8 \%)$ \\
Urinary tract & $3(12 \%)$ & $2(7.7 \%)$ \\
Mixed & $2(8 \%)$ & \\
\hline
\end{tabular}

Table 3. Therapy data.

\begin{tabular}{lcr}
\hline Therapy data & $\begin{array}{c}\text { P1 (N=25) } \\
\text { N (\%) }\end{array}$ & $\begin{array}{r}\text { P2 (N=26) } \\
\mathbf{N}(\mathbf{\%})\end{array}$ \\
\hline Therapy type & & \\
$\quad$ Empirical therapy & $16(64.0 \%)$ & $18(69.2 \%)$ \\
$\quad$ Specific therapy & $9(36.0 \%)$ & $8(30.8 \%)$ \\
Prior use of antibiotics & $21(84 \%)$ & $22(84.6 \%)$ \\
Association of antibiotics & $18(72 \%)$ & $16(61.5 \%)$ \\
Duration of therapy (days) & & \\
Mean \pm S.D. & $12.40 \pm 6.93$ & $11.85 \pm 6.96$ \\
\hline
\end{tabular}

For the analysis of the clinical outcomes the following were considered: 1 ) clinical cure as a resolution of the clinical signs of the infection, normalization of body temperature $(\mathrm{T}<$ $\left.37.2^{\circ} \mathrm{C}\right)$, decrease of the leucocytes $\left(<11.3 \times 10^{3} / \mathrm{mm}^{3}\right)$ and improvement of the patient's general state, 2 ) bacteriological cure as bacterial eradication up to 24 hours after the discontinuation of the therapy, 3) death and 4) transfer to another institution.

\section{Data Collection}

Data was collected from the medical and antimicrobial records in an instrument specifically designed and validated through a pilot model and with the assistance of a guideline manual for the completion of the form.

\section{Statistical Analysis}

Database was built in the software SPSS $₫$ for Windows $\AA$ PC version 10.0 for the recording and analysis of data.

The analysis carried out was of a descriptive nature where the nominal and ordinal variables were treated as frequencies and the continuous variables as mean, median and standard deviation.

The study was approved by the Research Ethics Committee of the Federal University of Bahia (UFBA).

\section{Results}

Of the 63 patients that used imipenem between May and December of 2006, 10 were lost and 4 excluded, leaving 49 patients, totaling 51 therapy courses. Table 1 describes the patients' general characteristics in the periods prior to (P1) and subsequent to (P2) the restructuring of the audit.

The average number of hospital admission was 561 and 420 patients/per month, the hospital occupancy rate was $80.0 \%$ and $65.0 \%$, and the hospital infection rate was $4.4 \%$ and $4.8 \%$, in $\mathrm{P} 1$ and $\mathrm{P} 2$, respectively. The number of immunosupressed patients was 6/25 (24.0\%) in P1 and 13/26 (50.0\%) in P2.

The total consumption of imipenem in the first period was 1,581 units and 1,041 units in the second period, with a reduction of $34.1 \%$, corresponding to 1.7 DDDs/100 bedsday in $\mathrm{P} 1$ and 1.6 DDDs/100 beds-day in P2.

Tables 2 and 3 summarize data on infection and therapy in both periods.

The appropriate use occurred in 14/25 (56\%) and 15/26 (57.7\%) of the therapy courses in P1 and P2, respectively. Table 4 displays the criteria used to determine the appropriate use of imipenem in the therapeutic indication requirement. In the antibiotics associations, 4/18 (22.2\%) and 1/16 (6.3\%) were considered inappropriate. In $\mathrm{P} 1$, there were two cases of inappropriate association with metronidazole, one with ampicillin and another with oxacilin. In P2, there was a case with metronidazole.

In P1, four antimicrobial schemes were modified: three for lack of clinical response and one for convulsion. In P2, eight antimicrobial schemes were modified: four for lack of clinical 
response, two for specific therapy adjustment and two for therapy de-escalation.

In the first period, $14 / 25$ (56\%) of the patients achieved clinical cure and 11/25 (44\%) deceased. In the second period, $15 / 26(57.7 \%)$ of the patients achieved clinical cure, 10/26 (38.5\%) deceased and 1/26 (3.8\%) discharged from antimicrobial use.

\section{Discussion}

The reduction of $34.1 \%$ in the number of units released does not represent a real decrease in the consumption of this antibiotic. Considering the comparison unit of DDDs/100 beds-day, the results were similar, due to reduction of the hospital occupancy rate in P2. A decrease of 30.6\% in the number of prescribed carbapenems and of $40 \%$ in the expenses with imipenem was discovered in other studies [16,17].

No differences were found between the periods with regards to the appropriate use of imipenem, and this similarity of results can be attributed to the low prohibitive nature of the audit. In Brazil, Resolution $n^{0} 1552$ of the Federal Council of Medicine grants the infectologist full powers to authorize the liberation of antibiotics [28]. However, due to the conduct adopted by the institution, the suggestions of the specialist in infectious diseases were often not accepted by the prescribers. Other factors leading to this outcome were: the absence of protocols ans of a policy for the dispensing of antibiotics of restricted use, the dispensing of the first doses of the antibiotic in the impossibility of obtaining an evaluation from the infectologist and the lack of data on hospital ecology, fundamental for the orientation of the initial empirical therapy which was at a compilation and publication stage during the period of the study.

There were no expressive differences among the groups, except for the immunosupression in $50.0 \%$ of the patients in $\mathrm{P} 2$, which can justify in certain cases the utilization of a broad spectrum therapy. There were also no differences in the hospital infection rate and the origin of the infection. The most targeted topography, in both periods, was the systemic one evidencing the seriousness of infections. In P2, in addition to the increase in the number of non-enteric Gram-negative bacilli isolated from $8 \%$ to $30.4 \%$, imipenem was the only therapeutic alternative for five isolated bacteria (one
Acinetobacter baumannii, three Klebsiella pneumoniae producing ESBL and one Klebsiella pneumoniae). In P1, only one isolated bacteria (Enterobacter) was sensitive to just imipenem. The therapy type, whether empirical or specific, was similar among the groups. The number of patients with a prior use of antibiotics also did not vary.

The appropriate use of imipenem occurred mainly in the empirical treatment of severe nosocomial infections, 10/14 (71.4\%) in P1 and 10/15 (66.7\%) in P2. This use is justifiable when based on the microbiological profile of the institution, since the real purpose of the audit is to reduce the indiscriminate use of the antibiotics, when choosing more cost-effective treatments, without, however, denying patients the right to the most suitable therapy, even if this conduct results in the increase of assistance costs.

The main cause of the inappropriate use of imipenem was the non-utilization of the more cost-effective alternative therapeutic in all the 11 cases in P1 and in 10/11 (90.9\%) of the cases in P2. In the latter period, the other cause of inappropriate use was the utilization of this antibiotic in an elderly patient, with extended stay in the intensive care unit (UCI) and with a diagnosis of lung sepsis caused by Acinetobacter baumannii and Enterobacter spp., having previously used imipenem for 17 days and that in the second culture the Acinetobacter baumannii was resistant to imipenem. The inappropriate associations occurred mainly with metronidazole and were used for a few days, the main reason being the non-suspension of this antibiotic following a therapy change. In P2, there was a reduction of 4 to 1 (75\%) in the irrational associations.

Higher figures regarding the appropriate use of imipenem of $65.2 \%$ and $70 \%$ were demonstrated in the utilization studies of this antibiotic, although these studies did not use a similar methodology [29,30].

An increase of 4 to 8 (50\%) in the change of therapy following the restructuring of the audit, above all, with regards to the adjustment of a specific therapy, or de-escalation of the initial therapy, is an indication that a specialist's support provides the medical team with a greater security in the treatment of serious infections. In the two periods no alterations were noted in the clinical outcomes of the patients. The limitation of this study refers to the reduced number of patients due to the short observation period, fact which did not allow finding significant differences among the periods.

Table 4. Analysis of the use of imipenem in the indication criterion.

\begin{tabular}{lcc}
\hline Indication of imipenem & $\mathbf{N}=\mathbf{2 5}$ & $\mathbf{N}=\mathbf{2 6}$ \\
\hline Appropriate & $\mathrm{P} 1(\mathrm{n}=14)$ & $\mathrm{P} 2(\mathrm{n}=15)$ \\
Empirical treatment of severe nosocomial infections* & $10(71.4 \%)$ & $10(66.7 \%)$ \\
Only alternative for the specific treatment of infections & $1(7.1 \%)$ & $5(33.3 \%)$ \\
Most cost-effective specific treatment & $3(21.4 \%)$ & - \\
Inappropriate & $\mathrm{P} 1(\mathrm{n}=11)$ & $\mathrm{P} 2(\mathrm{n}=11)$ \\
Existence of a more cost-effective therapeutic alternative & $11(100 \%)$ & $1090.9 \%)$ \\
Possibility of bacterial resistance & - & $1(9.1 \%)$ \\
\hline
\end{tabular}

* Based on the microbiological profile of the institution. 


\section{Conclusion}

The restructuring of the antimicrobial audit system did not reflect an increase of the appropriate use of imipenem in the four months following this intervention. Data of this study reinforce the institution' needs to adopt an antimicrobial audit of an educational nature with an intent to reduce the indiscriminate use of these drugs.

\section{Axknowledgements}

Ours special thanks to: Leonardo Kister, Márcio Galvão and Pablo Moura for their contributions during discussions of this study; Nadja Pereira de Souza, for her final paper in the Pharmaceutical Assistance Management Specialization Course of the Federal University of Bahia, under the orientation of Prof. Lúcia Noblat.

\section{References}

1. Balfour J., Bryson H., Brogden R. Imipenem/cilastatin. An update of its antibacterial activity, pharmacokinetics and therapeutic efficacy in the treatment of serious infections. Drugs 1996;51(1):99-136.

2. Rodloff A.C., Goldstein E.J., Torres A. Two decades of imipenem therapy. J Antimicrob Chemother 2006;58(5):916-29.

3. Fuchs D.F. Antibióticos Betalactâmicos. In: FUCHS, F.R; Wannmacher, L; Ferreira, M.B. Farmacologia Clinica: Fundamentos da Terapêutica Racional. $3^{\mathrm{a}} \mathrm{ed}$. Rio de Janeiro: Guanabara Koogan, 2004.

4. Hsueh P.R., Chen W.H., Luh K.T. Relationships between antimicrobial use and antimicrobial resistance in Gram-negative bacteria causing nosocomial infections from 1991-2003 at a university hospital in Taiwan. International journal of antimicrobial agents. 2005;26(6):463-72.

5. Lepper P.M., Grusa E., Reichl H. et al. Consumption of imipenem correlates with $\beta$-Lactam resistance in Pseudomonas aeruginosa. Antimicrob Agents Chemother 2002;46(9):2920-5.

6. Martínez J.A., Aguilar J., Almela M., et al. Prior use of carbapenems may be a significant risk factor for extended-spectrum betalactamasis-producing Escherichia coli or Klebsiella spp. in patients with bacteraemia. J Antimicrob Chemother 2006;58(5):1082-5.

7. Paterson D.L. Resistance in Gram-negative bacteria: Enterobacteriaceae. Am J Infect Control 2006;119(5):S20-8.

8. Powers J.H. Antimicrobial drug development - the past, the present, and the future. Clinical Microbiology and Infection 2004;10(Suppl.4):23-31.

9. Spellberg B., Powers J.H., Brass E.P., et al. Trends in antimicrobial drug development: implications for the future. Clin Infect Dis 2004;38(9):1279-86.

10. World Health Organization. The Rational Use of Drugs. Available at: <http://www.opas.org.br/medicamentos/temas>. Access on: September 02, 2006.

11. Slama T.G., Amin A., Brunton S.A., et al. A clinician's guide to the appropriate and accurate use of antibiotics: the Council for Appropriate and Rational Antibiotic Therapy (CARAT) criteria. Am J Med 2005;118(7A):1S-6S.

12. Aronson, J.K. Rational prescribing, appropriate prescribing. $\mathrm{Br} \mathrm{J}$ Clin Pharmacol 2004;57(3):229-30.
13. Oliveira M.G. Prescrição de Captopril Precedendo Elevação Pressórica em Pacientes Hospitalizados: Análise desta Conduta. Dissertação (Mestrado em Medicina e Saúde). Faculdade de Medicina da Bahia.Universidade Federal da Bahia, Salvador, 2007.

14. Goldmann D.A., Weinstein R.A., Wenzel R.P., et al. Strategies to Prevent and Control the Emergence and Spread of AntimicrobialResistant Microorganisms in Hospitals. A challenge to hospital leadership. JAMA. 1996;275(3):234-40.

15. Himmelberg C.J., Pleasants R.A., Weber D.J., et al. Use of antimicrobial drugs in adults before and after removal of a restriction policy. American journal of hospital pharmacy. 1991;48(6):1220-7

16. White A.C. Jr., Atmar R.L., Wilson J., et al. Effects of requiring prior authorization for selected antimicrobials: expenditures, susceptibilities, and clinical outcomes. Clin Infect Dis 1997;25(2):230-9.

17. Ozkurt Z., Erol S., Kadanali A., et al. Changes in antibiotic use, cost and consumption after an antibiotic restriction policy applied by infectious disease specialists. Japanese Journal of Infectious Diseases 2005;58(6):338-43.

18. Brink A.J., Feldman C., Grolman D.C., et al. Appropriate use of the carbapenems. S Afr Med J 2004;94:857-61.

19. Uptodate. Imipenem and cilastatin: Drug Information. Available at: < http:// www.uptodateonline.com>.Access on: September 02, 2006.

20. Sweetman S.C. (ed.). Martindale. The Complete Drug Reference.35 ed, Pharmaceutical Press, 2007.

21. Micromedex Healthcare Series, Thomson. Drug Information: [S.I]: Drugdex System, 2006. Acesso em: 02 de setembro de 2006. (Portal da CAPES).

22. American Society Of Health-System Pharmacists. AHFS Drug Information, 2005.

23. Paterson D.L. Recommendation for treatment of severe infections caused by Enterobacteriaceae producing extended-spectrum $\beta$ lactamases (ESBLs). Clin Microbiol Infect 2000;6:460-3.

24. Thomson K.S., Moland E.S. Cefepime, piperacillin-tazobactam, and the inoculum effect in tests with extended-spectrum betalactamase-producing Enterobacteriaceae. Antimicrob Agents Chemother 2001;45:3548.

25. Zimhony O., Chmelnitsky I., Bardenstein R., et al. Endocarditis caused by extended-spectrum-beta-lactamase-producing Klebsiella pneumoniae: emergence of resistance to ciprofloxacin and piperacillin-tazobactam during treatment despite initial susceptibility. Antimicrob Agents Chemother 2006;50:3179.

26. World Health Organization/ Who Collaborating Centre for Drug Statistics Methodology. Anatomical therapeutic chemical (ATC) classification index-including defined daily doses (DDDs) for plain substances. Oslo: WHO-Oslo. 1994b.

27. Clinical and Laboratory Standard Institute. Performance Standards for Antimicrobial Susceptibility Testing; Sixteenth Informational Supplement: Document M100-S16. CLSI, Wayne, PA, USA, 2006.

28. Conselho Federal De Medicina do Brasil. Resolução n ${ }^{\circ} 1.552$ de 20 de agosto de 1999.

29. Thuong M., Shortgen F., Zazempa V., et al. Appropriate use of restricted antimicrobial agents in hospitals: the importance of empirical therapy and assisted re-evaluation. J Antimicrob Chemother 2000;46(3):501-8.

30. Moreno T.G., Nacle I.J. Revisión de la utilización de medicamentos: imipenem/cilastatina en un hospital general básico. Farmacia Hospitalaria 1996:20(5):302-7. 